\title{
On the Existence of Korteweg - de Vries Solitons in Relativistic Hydrodynamics
}

\author{
D. A. Fogaça* \\ Instituto de Física, Universidade de São Paulo, C.P. 66318, 05315-970 São Paulo, SP, Brazil
}

Received on 29 September, 2006

\begin{abstract}
We study the conditions for the formation and propagation of Korteweg-de Vries solitons in relativistic fluid dynamics using an appropriate equation of state. The $\mathrm{KdV}$ equation is obtained from the relativistic version of the Euler and continuity equations.
\end{abstract}

Keywords: Solitary waves; Equation of state; Relativistic hydrodynamics

\section{INTRODUCTION}

In a previous work [1] we have studied the formation and propagation of Korteweg - de Vries (KdV) solitons in nuclear matter. We found that these solitary waves can indeed exist in the nuclear medium, provided that derivative couplings between the nucleon and the vector field are included. These couplings lead to an energy density which depends on the laplacian of the baryon density. For this class of equation of state (EOS), which is quite general as pointed out in [2], perturbations on the nuclear density can propagate as a pulse without dissipation.

During the analysis of several realistic nuclear equations of state, we realized that, very often the speed of sound $c_{s}^{2}$ is in the range $0.15-0.25$. Compared to the speed of light these values are not large but not very small either. This suggests that, even for slowly moving nuclear matter, relativistic effects might be sizeable. This concern alone would justify the extension of the formalism presented in [1], but there is another motivation for revisiting our early work. In the last years there has been increasing evidence for supersonic motion in the hot hadronic matter formed in collisions at RHIC. The most striking evidence is the observation of an approximate double bump structure recoiling against a jet, the socalled "away-side jet", measured by STAR [3]. This structure was interpreted as arising from a conical shock wave resulting from the supersonic motion of a fast parton through the hadronic medium [4]. This interesting conjecture gives us additional motivation for studying supersonic motion in relativistic hydrodynamics.

\section{RELATIVISTIC HYDROYNAMICS}

In this section we review the main expressions of one dimensional relativistic hydrodynamics. In natural units $(c=1)$ the velocity four vector $u^{v}$ is defined as:

$$
u^{0}=\gamma, \quad \vec{u}=\gamma \vec{v}
$$

where $\gamma$ is the Lorentz contraction factor given by:

$$
\gamma=\left(1-v^{2}\right)^{-1 / 2}
$$

* daviddif.usp.br
The velocity field of the matter is $\vec{v}=\vec{v}(t, x, y, z)$ and thus

$$
u^{v} u_{v}=1
$$

The energy-momentum tensor is, as usual, given by:

$$
T_{\mu v}=(\varepsilon+p) u_{\mu} u_{v}-p g_{\mu \nu}
$$

where $\varepsilon$ and $p$ are the energy density and pressure respectively. Energy-momentum conservation is ensured by:

$$
\partial_{v} T_{\mu}^{v}=0
$$

The projection of (5) onto a direction perpendicular to $u^{\mu}$ gives us the relativistic version of Euler equation $[5,6]$ :

$$
\frac{\partial \vec{v}}{\partial t}+(\vec{v} \cdot \vec{\nabla}) \vec{v}=-\frac{1}{(\varepsilon+p) \gamma^{2}}\left(\vec{\nabla} p+\vec{v} \frac{\partial p}{\partial t}\right)
$$

The relativistic version of the continuity equation for the baryon number is [5]:

$$
\partial_{v} j_{B}{ }^{v}=0
$$

Since $j_{B} v=u^{v} \rho_{B}$ the above equation reads

$$
\frac{\partial}{\partial t}\left(\rho_{B} \gamma\right)+\vec{\nabla} \cdot\left(\rho_{B} \gamma \vec{v}\right)=0
$$

The enthalpy per nucleon is given by [6]:

$$
d h=T d s+V d p
$$

where $\quad V=1 / \rho_{B} \quad$ is the specific volume. For a perfect fluid $(d s=0)$ the equation above becomes $d p=\rho_{B} d h$ and consequently:

$$
\vec{\nabla} p=\rho_{B} \vec{\nabla} h, \quad \frac{\partial p}{\partial t}=\rho_{B} \frac{\partial h}{\partial t}
$$

Inserting (10) in (6) we find:

$$
\frac{\partial \vec{v}}{\partial t}+(\vec{v} \cdot \vec{\nabla}) \vec{v}=-\frac{\rho_{B}}{(\varepsilon+p) \gamma^{2}}\left(\vec{\nabla} h+\vec{v} \frac{\partial h}{\partial t}\right)
$$

Recalling the Gibbs relation [7]:

$$
\varepsilon-T s+p=\mu_{B} \rho_{B}
$$

and considering the case where $T=0$ we obtain:

$$
\varepsilon+p=\mu_{B} \rho_{B}
$$


where $\varepsilon, p, \mu_{B}$ and $\rho_{B}$ are the energy density, pressure, baryochemical potential and baryon density respectively. Inserting (2) and (13) into (11) we obtain:

$$
\frac{\partial \vec{v}}{\partial t}+(\vec{v} \cdot \vec{\nabla}) \vec{v}=-\frac{\left(1-v^{2}\right)}{\mu_{B}}\left(\vec{\nabla} h+\vec{v} \frac{\partial h}{\partial t}\right)
$$

Since the enthalpy per nucleon may also be written as $[1,8]$ :

$$
h=\frac{\partial \varepsilon}{\partial \rho_{B}}
$$

it becomes clear that it is the equation of state that will determine the final form of (14).

We close this section comparing the relativistic and nonrelativistic versions of the Euler and continuity equations. The latter were presented in [1]

$$
\begin{gathered}
\frac{\partial \rho_{B}}{\partial t}+\vec{\nabla} \cdot\left(\rho_{B} \vec{v}\right)=0 \\
\frac{\partial \vec{v}}{\partial t}+(\vec{v} \cdot \vec{\nabla}) \vec{v}=-\left(\frac{1}{M}\right) \vec{\nabla} h
\end{gathered}
$$

and the former are (8) and (14)

$$
\begin{gathered}
\frac{\partial}{\partial t}\left(\rho_{B} \gamma\right)+\vec{\nabla} \cdot\left(\rho_{B} \gamma \vec{v}\right)=0 \\
\frac{\partial \vec{v}}{\partial t}+(\vec{v} \cdot \vec{\nabla}) \vec{v}=-\frac{\left(1-v^{2}\right)}{\mu_{B}}\left(\vec{\nabla} h+\vec{v} \frac{\partial h}{\partial t}\right)
\end{gathered}
$$

The two pairs are similar. The differences are only in the $\gamma$ factors and in the last term of (19), where the appearance of time derivative reflects the symmetry between space and time.

\section{THE KDV EQUATION}

In this section we follow the treatment developed in $[1,8,9]$ to obtain the Korteweg-de Vries equation in one dimension through the combination of (14) and (8). Concerning the hadronic medium we shall consider three cases: I) ordinary nuclear matter at baryon density $\rho_{0}$ for which the following saturation condition holds:

$$
\frac{\partial}{\partial \rho_{B}}\left(\frac{\varepsilon}{\rho_{B}}-M\right)_{\rho_{B}=\rho_{0}}=0
$$

In case II) we have ordinary and saturated nuclear matter described by the relativistic mean field model developed in [1] and called modified Quantum Hadrodynamics (MQHD). In case III) we have hadronic matter at arbitrary constant baryon density. This last choice is motivated by a future study of dense stars. We can represent these three systems with the equation of state:

$$
\varepsilon=\alpha_{1} \rho_{B}+\alpha_{2} \rho_{B}{ }^{2}+\alpha_{3} \rho_{B}{ }^{3}+\beta \rho_{B} \vec{\nabla}^{2} \rho_{B}
$$

where $\alpha_{i}$ and $\beta$ are constants.

This Ansatz is similar to the energy density used in $[1,8,9]$ and is consistent with the EOS obtained with the approach based on the density functional theory [2].

Using (21) to evaluate (15) and its derivatives we find:

$$
\vec{\nabla} h=\Phi \rho_{B} \vec{\nabla} \rho_{B}+\phi \vec{\nabla} \rho_{B}+\omega \vec{\nabla}\left(\vec{\nabla}^{2} \rho_{B}\right)
$$

and

$$
\frac{\partial h}{\partial t}=\Phi \rho_{B} \frac{\partial \rho_{B}}{\partial t}+\phi \frac{\partial \rho_{B}}{\partial t}+\omega \frac{\partial}{\partial t}\left(\vec{\nabla}^{2} \rho_{B}\right)
$$

where

$$
\begin{gathered}
\Phi \equiv \begin{cases}6 \alpha_{3} & \text { cases I and III } \\
\frac{3 M c_{s}^{2}}{\rho_{0}^{2}} & \text { case II }\end{cases} \\
\phi \equiv \begin{cases}2 \alpha_{2} & \text { case III } \\
-4 \alpha_{3} \rho_{0} & \text { case I } \\
\frac{-2 M c_{s}^{2}}{\rho_{0}} & \text { case II }\end{cases} \\
\omega \equiv \begin{cases}\beta & \text { cases I and III } \\
\frac{g_{V}{ }^{2}}{m_{V}} & \text { case II }\end{cases}
\end{gathered}
$$

We now repeat the steps developed in [1,9] and introduce dimensionless variables for the baryon density and velocity:

$$
\hat{\rho}=\frac{\rho_{B}}{\rho_{0}}, \quad \hat{v}=\frac{v}{c_{s}}
$$

We next define the "stretched coordinates" $\xi$ and $\tau$ as in [8-10]:

$$
\xi=\sigma^{1 / 2} \frac{\left(x-c_{s} t\right)}{R}, \quad \tau=\sigma^{3 / 2} \frac{c_{s} t}{R}
$$

where $R$ is a size scale and $\sigma$ is a small $(0<\sigma<1)$ expansion parameter chosen to be [10]:

$$
\sigma=\frac{\left|u-c_{s}\right|}{c_{s}}
$$

where $u$ is the propagation speed of the perturbation in question. We then expand (27) around the equilibrium values:

$$
\begin{gathered}
\hat{\rho}=1+\sigma \rho_{1}+\sigma^{2} \rho_{2}+\ldots \\
\hat{v}=\sigma v_{1}+\sigma^{2} v_{2}+\ldots
\end{gathered}
$$

After the expansion above (14) and (8) will contain power series in $\sigma$ (in practice we go up to $\sigma^{2}$ ). Since the coefficients in these series are independent of each other we get a set of equations, which, when combined, lead to the $\mathrm{KdV}$ equation for $\rho_{1}$ :

$$
\frac{\partial \rho_{1}}{\partial \tau}+\left(\frac{3}{2}+\frac{\Phi \rho_{0}^{2}}{2 \mu c_{s}^{2}}-c_{s}^{2}\right) \rho_{1} \frac{\partial \rho_{1}}{\partial \xi}+\left(\frac{\omega \rho_{0}}{2 \mu c_{s}^{2} R^{2}}\right) \frac{\partial^{3} \rho_{1}}{\partial \xi^{3}}=0
$$

with the condition

$$
\frac{\left(\Phi \rho_{0}+\phi\right) \rho_{0}}{\mu c_{s}^{2}}=1
$$




\section{SOLUTION OF THE KDV EQUATION}

The equation (32) has a well known soliton solution. We may rewrite the last equation back in the $x-t$ space obtaining a $\mathrm{KdV}$-like equation for $\hat{\rho}_{1}$ with the following analytical solitonic solution:

$$
\begin{aligned}
& \hat{\rho}_{1}(x, t)=\frac{3\left(u-c_{s}\right)}{c_{s}\left(\frac{3}{2}+\frac{\Phi \rho_{0}^{2}}{2 \mu c_{s}^{2}}-c_{s}^{2}\right)} \times \\
& \times \operatorname{sech}^{2}\left[\sqrt{\frac{\mu c_{s}\left(u-c_{s}\right)}{2 w \rho_{0}}}(x-u t)\right]
\end{aligned}
$$

where $\hat{\rho}_{1} \equiv \sigma \rho_{1}$.

This solution is a bump which propagates with speed $u$, without dissipation and preserving its shape.

The expressions given by (32), (33) and (34) depend on the choices given by (24), (25) and (26).

In case II (MQHD), the constraint (33) implies that $\mu=M$ and the general equation (32) becomes:

$$
\frac{\partial \rho_{1}}{\partial \tau}+\left(3-c_{s}^{2}\right) \rho_{1} \frac{\partial \rho_{1}}{\partial \xi}+\left(\frac{g_{V}^{2} \rho_{0}}{2 M m_{V}^{4} c_{s}^{2} R^{2}}\right) \frac{\partial^{3} \rho_{1}}{\partial \xi^{3}}=0
$$

with the solution given by:

$$
\begin{gathered}
\hat{\rho}_{1}(x, t)=\frac{3\left(u-c_{s}\right)}{c_{s}}\left(3-c_{s}{ }^{2}\right)^{-1} \times \\
\times \operatorname{sech}^{2}\left[\frac{m_{V}^{2}}{g_{V}} \sqrt{\frac{\left(u-c_{s}\right) c_{s} M}{2 \rho_{0}}}(x-u t)\right]
\end{gathered}
$$

As a consitency check we take the non-relativistic limit, which, in this case, means taking a small sound speed $c_{s}^{2} \rightarrow 0$. In this limit $\left(3-c_{s}{ }^{2}\right) \cong 3$ and (35) and (36) coincide the results previously obtained in [1]:

$$
\frac{\partial \rho_{1}}{\partial \tau}+3 \rho_{1} \frac{\partial \rho_{1}}{\partial \xi}+\left(\frac{g_{V}^{2} \rho_{0}}{2 M c_{s}^{2} m_{V}{ }^{4} R^{2}}\right) \frac{\partial^{3} \rho_{1}}{\partial \xi^{3}}=0
$$

and

$$
\hat{\rho}_{1}(x, t)=\frac{\left(u-c_{s}\right)}{c_{s}} \times
$$

$$
\times \operatorname{sech}^{2}\left[\frac{m_{V}^{2}}{g_{V}} \sqrt{\frac{\left(u-c_{s}\right) c_{s} M}{2 \rho_{0}}}(x-u t)\right]
$$

It is interesting to observe the supersonic nature of the solutions (36) and (38), which is manifest in the arguments of the square roots. As a final remark about (34) we notice that, for

$$
\frac{\Phi \rho_{0}^{2}}{2 \mu c_{S}^{2}}<c_{s}^{2}-\frac{3}{2}
$$

the solution (34) becomes negative and, in view of (30), can be interpreted as a rarefaction wave as in [8].

\section{CONCLUSIONS}

We have extended the results of our previous work [1], showing that it is possible to obtain the KdV solitons in relativistic hydrodynamics with an appropriate EOS. Taking the non-relativistic limit $\left(c_{s}^{2} \rightarrow 0\right)$ we were able to recover the previous results.

\section{Acknowledgments}

This work was partially financed by the Brazilian funding agencies CAPES, CNPq and FAPESP.
[1] D.A. Fogaça and F.S. Navarra, Phys. Lett. B 639, 629 (2006); Phys. Lett. B 645, 408 (2007).

[2] R.J. Furnstahl, B.D. Serot, and H.B. Tang, Nucl. Phys. A 615, 441 (1997); J.J. Rusnak and R.J. Furnstahl, Nucl. Phys. A 627, 495 (1997); R.J. Furnstahl and B.D. Serot, Nucl. Phys. A 671, 447 (2000)

[3] J. Adams et al., STAR Collab. Phys. Rev. Lett. 95, 152301 (2005); S.S. Adler et al., nucl-ex/0507004.

[4] T. Renk and J. Ruppert, Phys. Rev. C 73, 011901 (2006) and references therein.

[5] S. Weinberg, Gravitation and Cosmology, New York: Wiley, 1972;
[6] L. Landau and E. Lifchitz, Fluid Mechanics, Pergamon Press, Oxford, (1987).

[7] R. Reif, Fundamentals os statistical and thermal physics, New York: McGraw-Hill, 1965.

[8] A.Y. Abul-Magd, I. El-Taher, and F.M. Khaliel, Phys. Rev. C 45, 448 (1992)

[9] G.N. Fowler, S. Raha, N. Stelte, and R.M. Weiner, Phys. Lett. B 115, 286 (1982).

[10] R.C. Davidson, Methods in Nonlinear Plasma Theory, Academic Press, New York an London, 1972. 